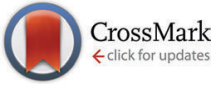

Cite this: Chem. Commun., 2016, 52,366

Received 2nd October 2015, Accepted 28th October 2015

DOI: $10.1039 / \mathrm{c5cc08223d}$

www.rsc.org/chemcomm

\section{Methyl tunnelling of adsorbed methoxy on alumina catalysts $\uparrow$}

\author{
Stewart F. Parker, ${ }^{\text {ab }}$ Victoria García Sakai, ${ }^{a}$ David Lennon, ${ }^{\text {bc }}$ Alice Jackson, ${ }^{d}$ \\ Mark R. Johnson ${ }^{d}$ and Upali A. Jayasooriya ${ }^{e}$
}

The aim of this paper is to investigate whether the methyl group of the adsorbed methoxy intermediate on industrial grade alumina catalysts undergoes rotational tunnelling on the wavenumber energy scale. The data show that this is clearly the case for a fraction of the methyl groups and potentially allows the subtle intermolecular interactions between adsorbed species and catalyst to be probed through the exponential dependence of the tunnel frequency on the rotational potential.

Heterogeneous catalysis is a key economic driver in advanced nations. ${ }^{1}$ It is integral to processes that range from crude oil refining to fine chemical and pharmaceutical production. ${ }^{2}$ Hence there is enormous interest and effort in optimising catalyst performance.

Alumina is ubiquitous in heterogeneous catalysis, where it is used as an actual catalytic material or as a catalyst support material. ${ }^{3}$ In fact, due to its general availability, structural stability and ability to be prepared in a variety of pore size distributions, alumina is the most widely used catalyst support material.

The interaction of methanol with alumina is important in the industrial manufacture of methyl chloride, where methanol and hydrogen chloride are combined over high surface area alumina catalysts:

$$
\mathrm{CH}_{3} \mathrm{OH}+\mathrm{HCl} \rightarrow \mathrm{CH}_{3} \mathrm{Cl}+\mathrm{H}_{2} \mathrm{O}
$$

\footnotetext{
${ }^{a}$ ISIS Facility, STFC Rutherford Appleton Laboratory, Chilton, Didcot, Oxfordshire OX11 oQX, UK. E-mail: stewart.parker@stfc.ac.uk

${ }^{b}$ UK Catalysis Hub, Research Complex at Harwell, STFC Rutherford Appleton Laboratory, Chilton, Didcot, Oxfordshire OX11 OQX, UK

${ }^{c}$ School of Chemistry, Joseph Black Building, University of Glasgow, Glasgow G12 8QQ, UK

${ }^{d}$ Institut Laue-Langevin, 71 Avenue des Martyrs, CS 20156, F- 38042 Grenoble Cedex 9, France

${ }^{e}$ School of Chemistry, Faculty of Science, University of East Anglia,

Norwich Research Park, Norwich, Norfolk, NR4 7TJ, UK

$\dagger$ Dedicated to the memory of Professor Norman Sheppard FRS 1922-2015.

\# Electronic supplementary information (ESI) available: Experimental details (sample preparation and instrumentation), computational methods and assignment of the spectrum of adsorbed methoxy. See DOI: 10.1039/c5cc08223d
}

We have previously investigated this reaction with infrared and inelastic neutron scattering (INS) spectroscopies. ${ }^{4-6}$ The key intermediate is adsorbed methoxy, $\mathrm{CH}_{3} \mathrm{O} \equiv$.

"Rotational tunnelling describes the phenomenon of the librational states of a molecule whose rotating atoms are indistinguishable, e.g., all protons, being multiplets. The splitting between the substates is called tunnel splitting", ${ }^{7}$ Thus for a methyl group, the protons essentially 'circulate' between the equivalent positions. If the protons are labelled 1, 2, 3 then the methyl group rotation $123 \rightarrow 231 \rightarrow 312 \rightarrow$ etc.. between the equivalent orientations has a tunnel frequency, $\omega_{\mathrm{t}}$, which is largely determined $^{7}$ by the amplitude of the rotational potential, $V$ :

$$
\hbar \omega_{\mathrm{t}}=\beta \exp \left(-\alpha \sqrt{\frac{|V|}{B}}\right)
$$

where $B$ is the rotational constant, $\beta \approx 2 B, \alpha \approx 1$. The exponential dependence of the tunnel frequency on the rotational potential is illustrated in Fig. 1, which shows the tunnelling and libration frequencies obtained by solving Schrödinger's equation for rotational motion in a 3-fold potential, of form $\cos (3 \phi)$, where $\phi$ is the methyl group rotation angle and $B=5.25 \mathrm{~cm}^{-1}$ : this

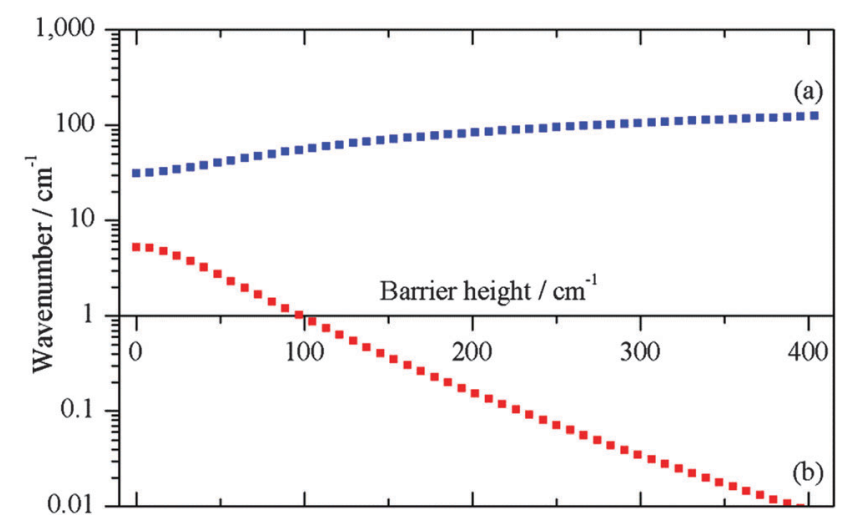

Fig. 1 The librational (a) and tunnelling (b) energies calculated as a function of the barrier height, $V$, for a 3 -fold rotational potential. 


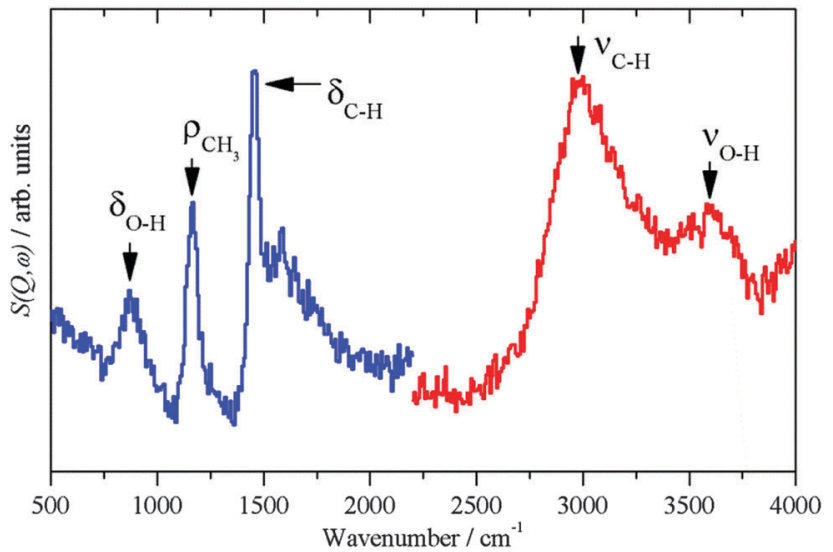

Fig. 2 INS spectrum of methoxy chemisorbed on $\eta$-alumina.

makes tunnelling spectroscopy uniquely sensitive to the weak interactions governing rotation.

To our knowledge, this is the first time tunnelling has been looked for on a real catalyst, although Larese et al. have extensively studied physisorbed methane on (essentially) single crystal MgO. ${ }^{8}$

Fig. 2 shows the INS spectrum of $\eta$-alumina after reaction with methanol at $373 \mathrm{~K}$. The peaks at 2990, 1456 and $1164 \mathrm{~cm}^{-1}$ are assigned as the $\mathrm{C}-\mathrm{H}$ stretch, bend and rock of the methyl group and confirms the presence of chemisorbed methoxy. ${ }^{5}$ The peaks at 3600 and $872 \mathrm{~cm}^{-1}$ are assigned as the $\mathrm{O}-\mathrm{H}$ stretch and bend of residual hydroxyls on the alumina. ${ }^{4}$

Fig. 3 shows the quasielastic neutron scattering (QENS) spectra at $2 \mathrm{~K}$ of clean $\gamma$-alumina and after reaction with methanol at $373 \mathrm{~K}$ to generate methoxy. It is apparent that there is a marked broadening of the central peak showing that there is additional motion in the system. Comparison of methoxy on $\eta$ - and $\gamma$-alumina, Fig. 4, shows both materials behave similarly, the greater intensity of the $\eta-\mathrm{Al}_{2} \mathrm{O}_{3}$ spectrum arising partly because it has a larger surface area and a greater density of active sites for strong chemisorption, hence the quantity of adsorbed methoxy is greater for $\eta-\mathrm{Al}_{2} \mathrm{O}_{3}$ than for $\gamma-\mathrm{Al}_{2} \mathrm{O}_{3}$. As discussed below, the fraction of sites with low enough rotational barriers

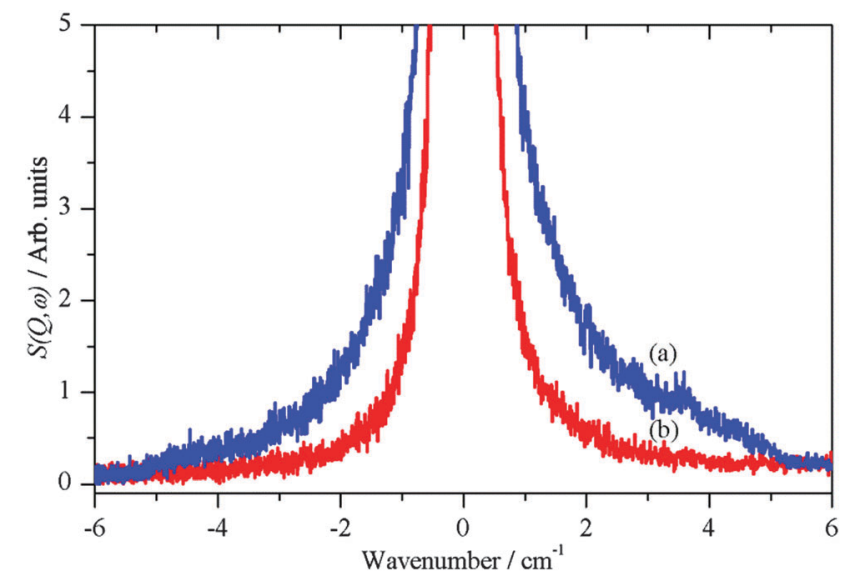

Fig. 3 QENS spectra at $2 \mathrm{~K}$ of $\gamma$-alumina (a) after reaction with methanol to generate methoxy (blue) and (b) clean (red).

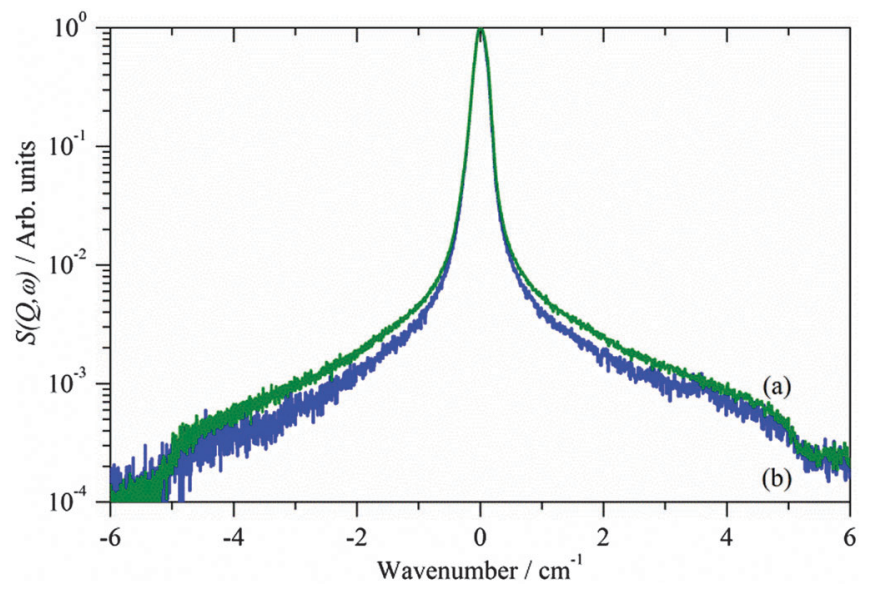

Fig. 4 Comparison of the QENS spectra at $2 \mathrm{~K}$ of (a) $\eta$-alumina (olive) and (b) $\gamma$-alumina (blue) after reaction with methanol to generate methoxy.

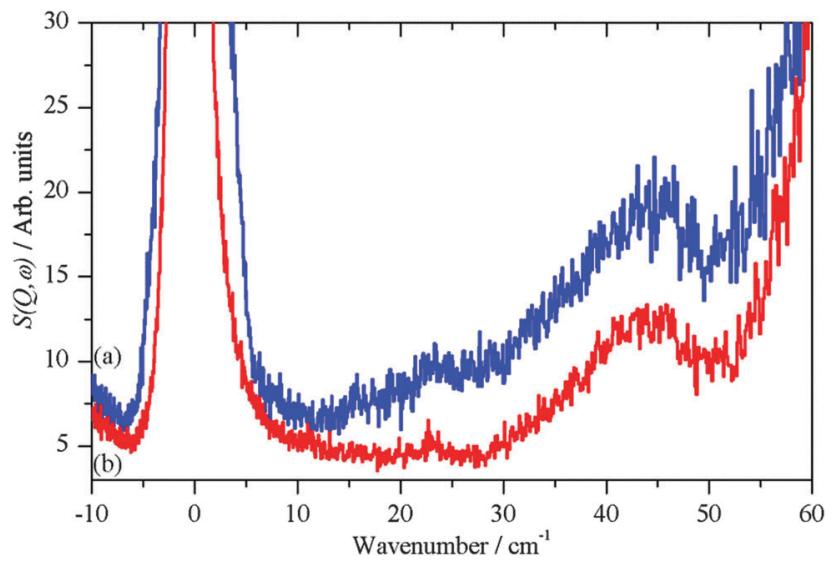

Fig. 5 QENS spectra at $2 \mathrm{~K}$ in an extended spectral range of $\gamma$-alumina (a) after reaction with methanol to generate methoxy (blue) and (b) clean (red).

to give a tunnelling signal in this energy range must also be taken into consideration.

As shown in Fig. 5a, in addition to the apparent quasielastic broadening, there is an additional feature at $\sim 24 \mathrm{~cm}^{-1}$. A feature at $45 \mathrm{~cm}^{-1}$, is also present in the clean $\gamma$-alumina, Fig. $5 b$, the same features are seen for $\eta-\mathrm{Al}_{2} \mathrm{O}_{3}$.

The intensity of neutron scattering spectra is strongly dependent on the incoherent cross section, $\sigma$, of the scattering atoms. This property is both element and isotope dependent and in particular $\sigma\left({ }^{1} \mathrm{H}\right)=80.26$ barn $\left(1\right.$ barn $\left.=1 \times 10^{-28} \mathrm{~m}^{2}\right)$ while $\sigma\left({ }^{2} \mathrm{H}\right)=2.05$ barn. Thus, by using selectively deuterated methanol, it is possible to determine the origin of the scattering. Fig. 6a shows the results for the reaction of $\mathrm{CH}_{3} \mathrm{OD}$ and Fig. $6 \mathrm{~b}$ for $\mathrm{CD}_{3} \mathrm{OH}$ with $\eta$-alumina. While the profile from reaction with $\mathrm{CH}_{3} \mathrm{OD}$ (trace (a)) is very similar to those obtained with $\mathrm{CH}_{3} \mathrm{OH}$, Fig. 3 and 5, reaction with $\mathrm{CD}_{3} \mathrm{OH}$ (trace (b)) results in almost no additional scattering, which demonstrates that all the features are due to methoxy, $\mathrm{OH}$ produced by the reaction has no effect in this energy region. (Chemisorption of methoxy and methoxy- $\mathrm{D}_{3}$ was confirmed by INS spectroscopy, see Fig. S3 and S4 of the ESI 

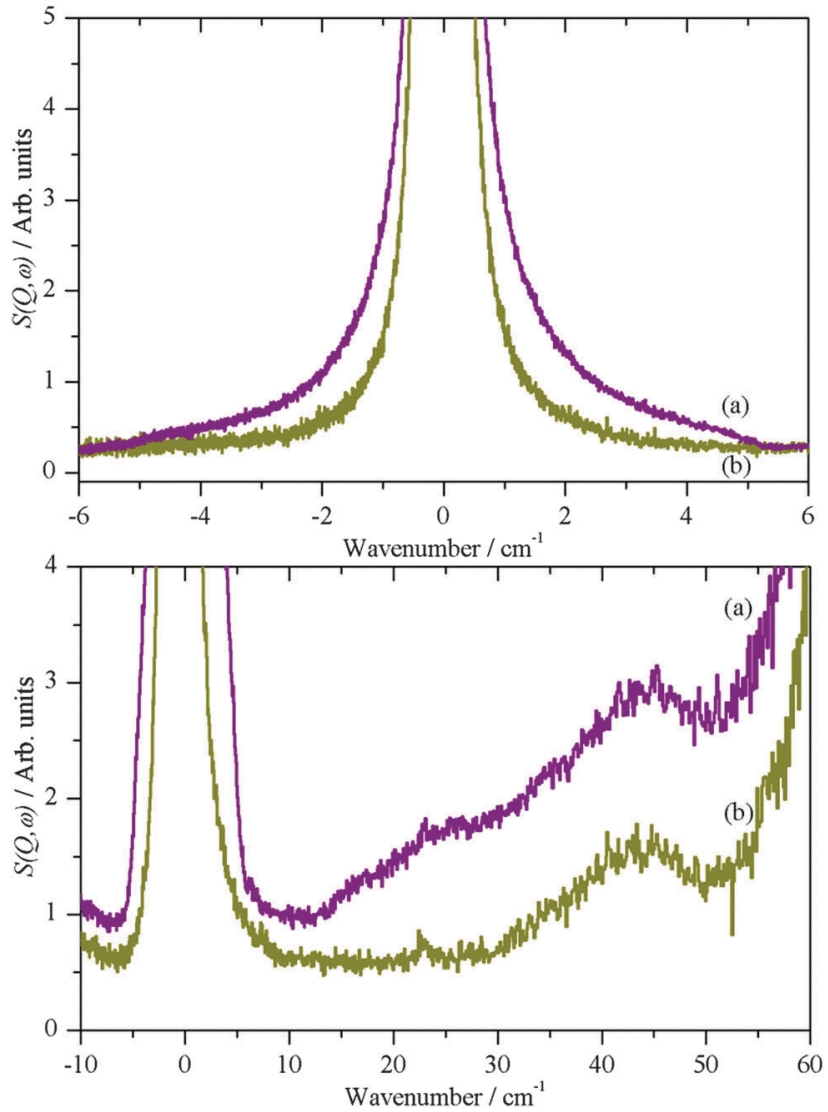

Fig. 6 QENS spectra at $2 \mathrm{~K}$ in a narrow (upper) and a wide (lower) energy range of the reaction products of selectively deuterated methanol with

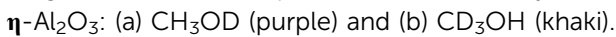

The data clearly show that chemisorption of methoxy on either alumina results in significant changes in the very low energy region. The peak at $\sim 24 \mathrm{~cm}^{-1}$ is assigned to surface modes of the alumina that acquire intensity because they result in motion of the methoxy - 'riding modes'. A calculation ${ }^{5}$ at the $\Gamma$-point of methoxy on $\eta$-alumina predicts such modes around $16 \mathrm{~cm}^{-1}$, non-zone centre modes (which are observable by INS spectroscopy) are also a possible assignment. The feature at $45 \mathrm{~cm}^{-1}$, which is present in all the spectra before and after reaction with methanol must be assigned to a bulk alumina phonon.

The broadening present in Fig. 3-5a would usually be described as quasielastic scattering due to diffusional processes, however, this possibility can be discarded because it is still present at $2 \mathrm{~K}$, see Fig. 3. Rather, we assign it as the signature of methyl tunnelling of the adsorbed methoxy species. The Lorentzian broadening is indicative of a distribution of adsorption sites giving rise to a continuous range of rotational potentials. From the data shown in Fig. 1, tunnelling resolved in the energy range from 0.5 to $6 \mathrm{~cm}^{-1}$ corresponds to rotational barriers, $V<120 \mathrm{~cm}^{-1}$. The fraction of sites with such low barriers can be estimated from the librational spectrum of the methyl groups shown in Fig. 7. Assuming, to a first approximation, that libration frequency varies linearly with $V$ and that the inelastic structure factor for librations is independent of their frequency, the spectrum, up to $\sim 200 \mathrm{~cm}^{-1}$, reflects directly the

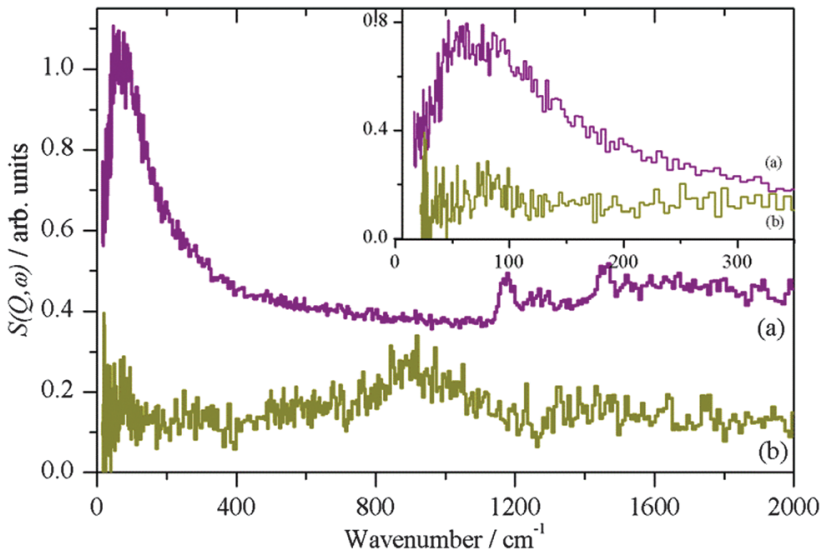

Fig. 7 INS spectra at $20 \mathrm{~K}$ the product of the reaction of selectively deuterated methanol with $\eta-\mathrm{Al}_{2} \mathrm{O}_{3}$ : (a) $\mathrm{CH}_{3} \mathrm{OD}$ (purple) and (b) $\mathrm{CD}_{3} \mathrm{OH}$ (khaki). The inset shows the librational region on an expanded scale.

distribution of rotational potentials. The tunnelling data corresponds to libration frequencies below $60 \mathrm{~cm}^{-1}$, which represents $\sim 15 \%$ of the spectral weight, and therefore absorbed species, up to $200 \mathrm{~cm}^{-1}$. This result is supported by the relative areas of the quasielastic contribution and the total scattering.
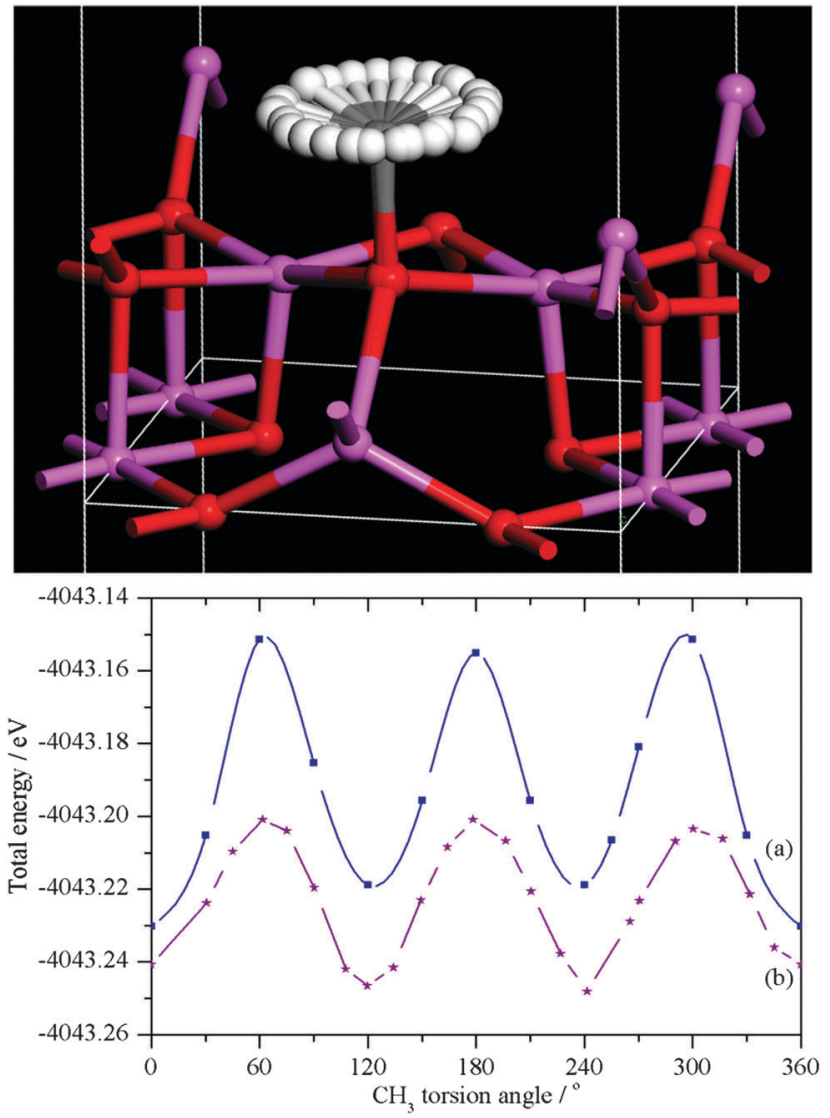

Fig. 8 Upper: Model of methoxy on $\eta-\mathrm{Al}_{2} \mathrm{O}_{3}$ used to generate the rotational potential. (Magenta $=\mathrm{Al}$, red $=\mathrm{O}$, grey $=\mathrm{C}$, white $=\mathrm{H}$ ). Lower: Rotational potential when: (a) the methyl group is held rigid and (b) when it is allowed to relax. Computational details are given in the ESI.‡ 
For both $\mathrm{CH}_{3} \mathrm{OH}$ and $\mathrm{CH}_{3} \mathrm{OD}$ reacted with $\eta-\mathrm{Al}_{2} \mathrm{O}_{3}$, we find $\sim 15 \%$ undergoes rotational tunnelling.

It has been proposed that there are four types of Lewis acid sites on alumina. ${ }^{6}$ Local structure variation around each of these sites would give rise to a distribution of rotational barriers and therefore libration and tunnelling frequencies. According to the assumptions proposed above, the distribution of libration frequencies in Fig. 7 could therefore be fitted with, for example, four independent Gaussian distributions. Clearly the data does not allow this to be done in an unambiguous way, without additional information. Based on a model system of methoxy on $\eta-\mathrm{Al}_{2} \mathrm{O}_{3}$, see Fig. 8, which was used previously to analyse INS spectra in a broad frequency range, we have calculated the potential barrier ( $V$ in eqn (2)) as a function of the rotation of the methyl group. If the methyl group is kept rigid the barrier is $484-565 \mathrm{~cm}^{-1}$ (60-70 meV) (dark blue curve (a) in Fig. 8 lower panel), if the methyl group is allowed to relax the barrier is reduced to $\sim 320 \mathrm{~cm}^{-1}(\sim 40 \mathrm{meV}$ ) (purple curve (b) in Fig. 8 lower panel). Using the relaxed potential, the librational and tunnelling energies are calculated as a function of the barrier height, as shown in Fig. 1. The tunnel splitting is $0.025 \mathrm{~cm}^{-1}$, that is inside the elastic line and therefore not resolved in the quasielastic data, and the librational frequency is $110 \mathrm{~cm}^{-1}$, just beyond the maximum $\sim 75 \mathrm{~cm}^{-1}$ of the broad distribution of libration frequencies shown in Fig. 7.

In conclusion, we have shown unequivocally that the methyl group of the adsorbed methoxy intermediate on industrial grade alumina catalysts undergoes rotational tunnelling in the wavenumber energy range. The observed signal corresponds to about $15 \%$ of the total amount of methoxy being adsorbed on sites with low barriers for the methyl group rotation. 85\% of adsorbed methoxy is on sites with higher rotational potentials for the methyl group, giving rise to the elastic signal in the tunnelling spectra and the distribution of libration frequencies up to $\sim 200 \mathrm{~cm}^{-1}$. Calculation of the rotational potential for an established model of an adsorption site of methoxy on alumina confirms the existence of sites with high rotational potentials. A comprehensive computational study is now required to identify and characterise energetically the full set of adsorption sites on alumina. Calculations establish the link between structure and excitations and demonstrate how the tunnelling data presented here can be used to probe the subtle intermolecular interactions between adsorbed species and catalyst. Using other backscattering spectrometers, with energy resolution of the order of $0.01 \mathrm{~cm}^{-1}$, the observable tunnelling spectrum could be sampled to lower energies.

This work was supported by the UK Catalysis Hub (EPSRC grant no. EP/K014714/1). We thank the STFC Rutherford Appleton Laboratory for access to neutron beam facilities. Experimental assistance and helpful discussions with Dr Franz Demmel and Dr Eugene A. Goremychkin (ISIS Facility) are gratefully acknowledged.

\section{Notes and references}

1 M. Morbidelli, A. Gavriilidis and A. Varma, Catalyst Design, Cambridge University Press, Cambridge, 2001, p. 1.

2 H. J. Arpe, Industrial Organic Chemistry, Wiley-VCH, Weinheim, 3rd edn, 2010.

3 R. J. Farrauto and C. H. Bartholomew, Fundamentals of Industrial Catalytic Processes, Blackie, London, 1997, p. 60.

4 A. R. McInroy, D. T. Lundie, J. M. Winfield, C. C. Dudman, P. Jones, S. F. Parker and D. Lennon, Catal. Today, 2006, 114, 403.

5 A. R. McInroy, D. T. Lundie, J. M. Winfield, C. Dudman, P. Jones, S. F. Parker, J. W. Taylor and D. Lennon, Phys. Chem. Chem. Phys., 2005, 7, 3093.

6 D. Lennon and S. F. Parker, Acc. Chem. Res., 2014, 47, 1220-1227.

7 M. Prager and A. Heidemann, Chem. Rev., 1997, 97, 2934.

8 J. Z. Larese, T. Arnold, A. Barbour and L. R. Frazier, Langmuir, 2009, 25, 4078-4083. 